\title{
Molecular Docking and Dynamics Investigations for Identifying Potential Inhibitors of the 3-Chymotrypsin-like Protease of SARS-CoV-2: Repurposing of Approved Pyrimidonic Pharmaceuticals for COVID-19 Treatment
}

\author{
Amin Osman Elzupir
}

check for updates

Citation: Elzupir, A.O. Molecular Docking and Dynamics Investigations for Identifying Potential Inhibitors of the 3-Chymotrypsin-like Protease of SARS-CoV-2: Repurposing of Approved Pyrimidonic

Pharmaceuticals for COVID-19 Treatment. Molecules 2021, 26, 7458 https://doi.org/10.3390/ molecules26247458

Academic Editors: Giovanni Ribaudo and Laura Orian

Received: 30 September 2021 Accepted: 29 November 2021 Published: 9 December 2021

Publisher's Note: MDPI stays neutral with regard to jurisdictional claims in published maps and institutional affiliations.

Copyright: (C) 2021 by the author. Licensee MDPI, Basel, Switzerland. This article is an open access article distributed under the terms and conditions of the Creative Commons Attribution (CC BY) license (https:// creativecommons.org/licenses/by/ $4.0 /)$.
College of Science, Deanship of Scientific Research, Imam Mohammad Ibn Saud Islamic University (IMSIU), Riyadh 11623, Saudi Arabia; aoalamalhuda@imamu.edu.sa

\begin{abstract}
This study demonstrates the inhibitory effect of 42 pyrimidonic pharmaceuticals (PPs) on the 3-chymotrypsin-like protease of SARS-CoV-2 (3CL pro ) through molecular docking, molecular dynamics simulations, and free binding energies by means of molecular mechanics-Poisson Boltzmann surface area (MM-PBSA) and molecular mechanics-generalized Born surface area (MM-GBSA). Of these tested PPs, 11 drugs approved by the US Food and Drug Administration showed an excellent binding affinity to the catalytic residues of 3CL pro of His41 and Cys145: uracil mustard, cytarabine, floxuridine, trifluridine, stavudine, lamivudine, zalcitabine, telbivudine, tipiracil, citicoline, and uridine triacetate. Their percentage of residues involved in binding at the active sites ranged from 56 to 100 , and their binding affinities were in the range from $-4.6 \pm 0.14$ to $-7.0 \pm 0.19 \mathrm{kcal} / \mathrm{mol}$. The molecular dynamics as determined by a $200 \mathrm{~ns}$ simulation run of solvated docked complexes confirmed the stability of PP conformations that bound to the catalytic dyad and the active sites of $3 C^{\text {pro }}$. The free energy of binding also demonstrates the stability of the PP-3CL ${ }^{\text {pro }}$ complexes. Citicoline and uridine triacetate showed free binding energies of -25.53 and $-7.07 \mathrm{kcal} / \mathrm{mol}$, respectively. Therefore, I recommend that they be repurposed for the fight against COVID-19, following proper experimental and clinical validation.
\end{abstract}

Keywords: coronavirus SARS-CoV-2; COVID-19; 3-chymotrypsin-like protease; pyrimidonic pharmaceuticals; molecular dynamics simulations; binding free energy

\section{Introduction}

Over a year has passed since the COVID-19 pandemic began. Some vaccines, such as those by Pfizer and Moderna, and some drugs, such as remdesivir, have been approved for use in therapy. The efforts by governments, health organizations, and other sectors to stem the alarmingly increasing numbers of deaths and cases were unprecedented [1-6]. However, SARS-CoV-2 continues to threaten the world, with over four million deaths and 227 million cases as of 16 September 2021 (https: / / www.worldometers.info/coronavirus / accessed on 27 November 2021). COVID-19 was declared a pandemic by the World Health Organization on 11 March 2020. Today, the new SARS-CoV-2 virus, the causative agent of COVID-19, has been detected in almost every country on the planet [5,7-9].

Coronaviruses are positive-stranded RNA viruses with the largest viral genomes ever known, ranging from 16 to $32 \mathrm{~kb}$. The 3-chymotrypsin-like protease (3CL ${ }^{\text {pro }}$ ) produced by SARS-CoV-2 is a cysteine protease encoded as nonstructural protein 3 in the polyprotein. $3 \mathrm{CL}^{\text {pro }}$ is responsible for the cleavage of 11 specific sites of polyproteins (pp1a, pp1ab) produced by the $229 \mathrm{E}$ gene. These polyproteins are involved in the production of a functional polypeptide essential for viral replication and transcription. Further, the specificity of $3 \mathrm{CL}^{\text {pro }}$ is dissimilar to that of human host-cell protease. Thus, $3 \mathrm{CL}^{\text {pro }}$ has become the focus of drug repurposing and development programs to combat the COVID-19 pandemic [10-13]. 
Recent and ongoing research has reported that some pharmaceutical, synthetic, and natural products can act as $3 \mathrm{CL}^{\text {pro }}$ inhibitors or against SARS-CoV-2 in general. These include selenium-containing heterocyclic compounds, chloroquine phosphate, indinavir, darunavir, lopinavir, eravacycline, naproxen, salix cortex, antioxidants, chiral phytochemicals from Opuntia ficus-indica, elbasvir, valrubicin, favipiravir isoflavone, and myricitrin [6,14-22]. Although some of these have entered human clinical trials or were even approved, more studies are still needed. The importance of the pyridone ring was highlighted in synthetic materials and drugs containing pyridone [11,23]. The pyrimidone ring has the exact shape of pyridine but is more functionalized and electron-deficient. Herein, we screened the inhibitory activity of 42 approved pyrimidonic pharmaceuticals (PPs) against $3 \mathrm{CL}^{\text {pro }}$ using a combination of molecular docking analyses, molecular dynamics simulations, and calculations of the MM-PBSA and MM-GBSA binding free energies. The sites of action of active inhibitors were investigated, discussed, and explored.

\section{Materials and Methods}

\subsection{The Pyrimidonic Pharmaceuticals (PPs)}

The PPs were selected using the search engine of the drug bank database. The search uncovered 46 PPs; the pharmaceuticals containing caffeine were entirely excluded as all except enprofylline have previously been studied. In addition, the macropolymeric drug mipomersen, drugs composed of a mixture of drugs, and withdrawn drugs were not included in this study. The chosen drugs were classified into four categories according to their structures. 1PPs have only one heterocycle, 2aPPs have two, 2bPPs have two heterocycles with a pyrimidone ring having an extra carbonyl group, and 3PPs have three or more heterocycles (Table 1).

Table 1. Structures of pyrimidonic pharmaceuticals and their classification according to the number of rings.

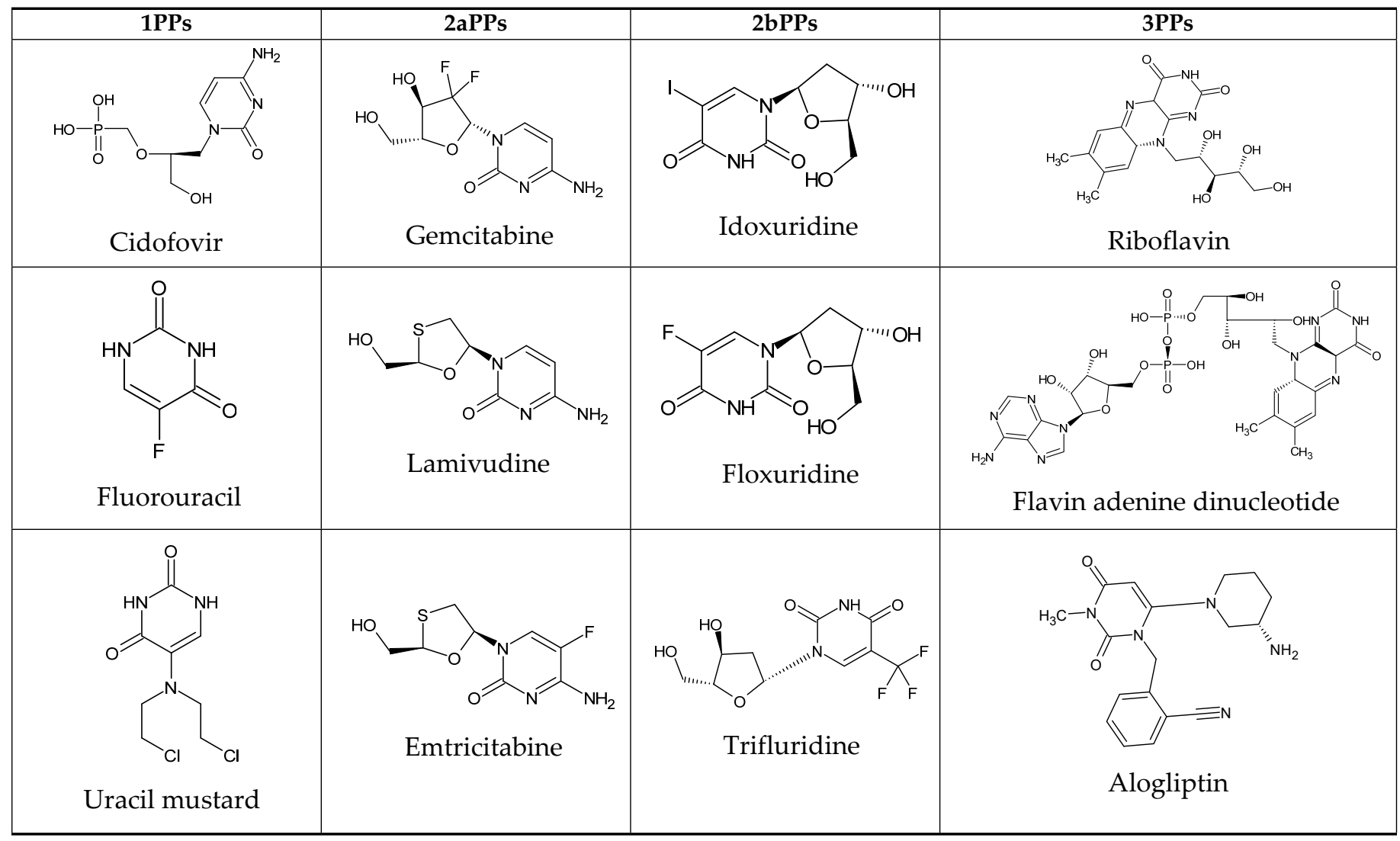


Table 1. Cont.

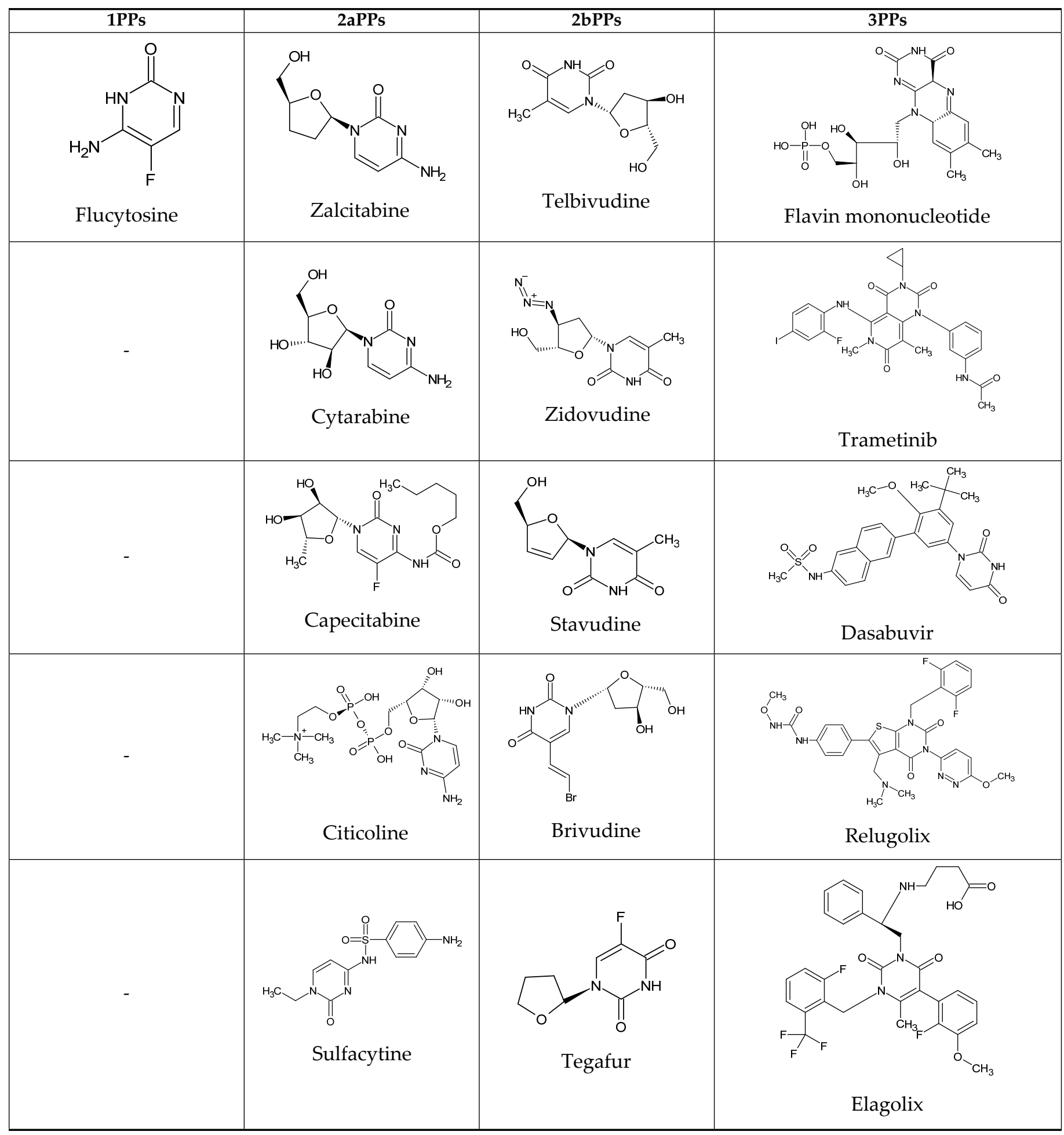


Table 1. Cont.

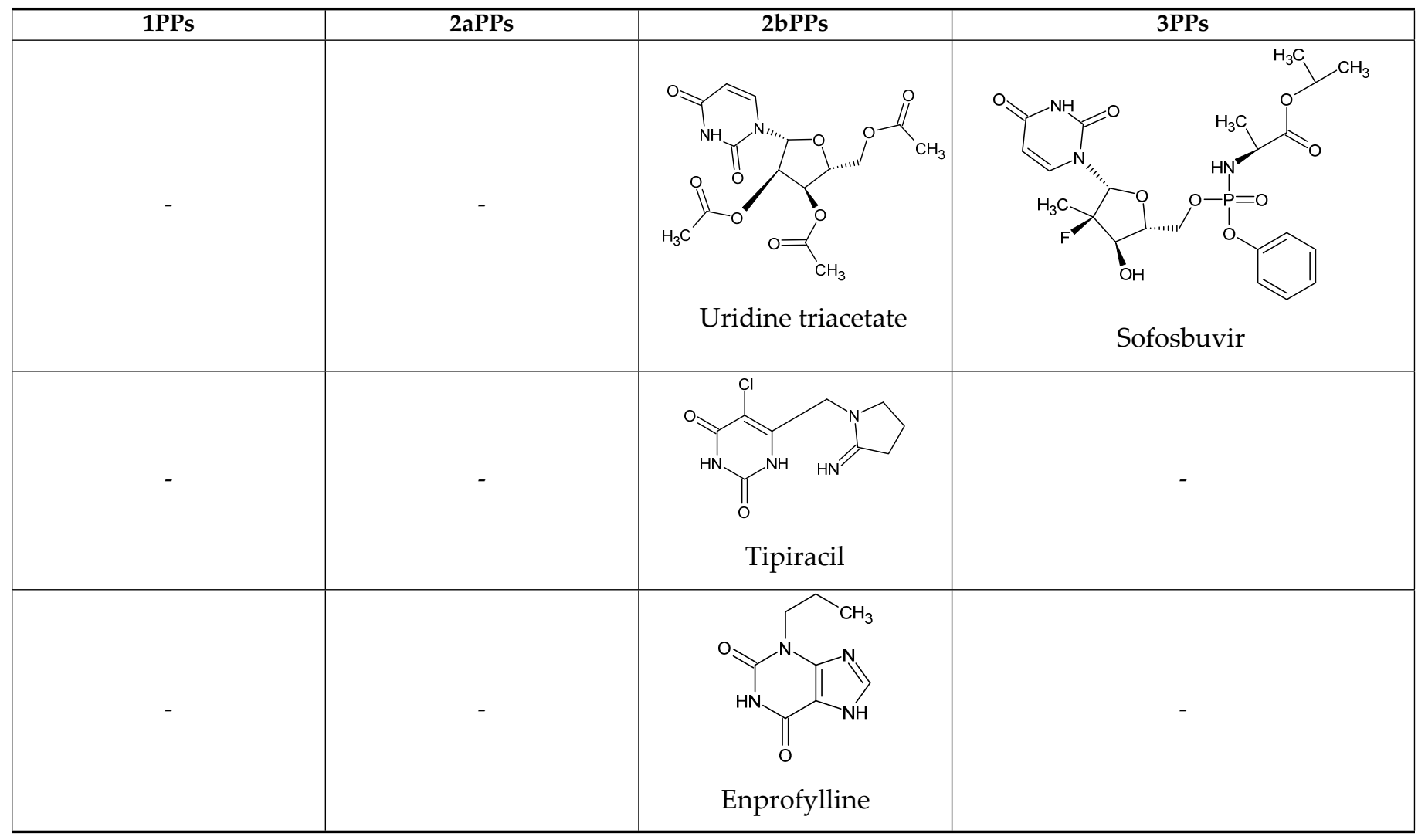

\subsection{Generation and Energy Minimization of the PPs and $3 C L^{\text {pro }}$}

The 3D structures of the selected PPs were downloaded from the PubChem website as SDF files; their energy was minimized for 10,000 steepest descent steps at 5000 conjugate gradient steps using antechamber plugin UCSF Chimera [24,25]. For alogliptin, the 3D structure was obtained by utilizing OpenBabel converter tools and ChemSkech [26]. The crystal structure of SARS-CoV-2 3CL pro was obtained from the Protein Data Bank database website (PDB ID: 6Y2E). For analysis, water was removed from the $3 \mathrm{CL}^{\text {pro }}$ structure, and the energy was then minimized for 1000 steepest descent steps at 20 conjugate gradient steps.

\subsection{Molecular Docking}

Blind molecular docking experiments were performed using the AutoDock Vina tool implemented with the interactive visualization and analysis program UCSF Chimera. The default parameter values were adopted with a grid box $(-15 \times-25 \times 15) \AA$, centered at $(35,65,65) \AA$. The predicted affinity values of the score were observed using the View Dock tool. The binding between ligands and $3 \mathrm{CL}^{\text {pro }}$ active sites and the images were processed and visualized using UCSF Chimera [24-28].

\subsection{Molecular Dynamics Simulations}

MD simulations were performed as previously described [29]. The PP ligands were separated from the docked complexes using UCSF Chimera. The missed hydrogens were added and saved as PDB files using AMBER's large-structure serial numbering. Topology files and parameters of the receptor and the ligands were made using leap and antechamber of Amber Tools 21 [30,30], utilizing Amber force fields of GAFF2 [31] and ff14SB [32] to assign inhibitors and $3 \mathrm{CL}^{\text {pro }}$ structure, respectively. The systems were solvated with TIP3P water molecules [33] and were neutralized via sodium ions. Subsequently, molecular dynamics (MD) simulations were performed by means of the Nanoscale Molecular Dynamics 
(NAMD) Simulation 2.6 program [34]. Each system was minimized for 1 ps at $273.15 \mathrm{~K}$ using the NVE ensemble. The temperature was gradually increased to $310 \mathrm{~K}$ using the NVT ensemble in a protocol consisting of 1600 minimization steps. Then, each system was minimized for $10 \mathrm{ps}$ at $310 \mathrm{~K}$ followed by $200 \mathrm{~ns}$ of MD simulation control using the NVT ensemble at $310 \mathrm{~K}$ and a time step of $2 \mathrm{fs}$. In order to calculate electrostatic interactions, the particle mesh Ewald process and periodic boundary conditions were applied $[35,36]$. The root-mean-square fluctuation (RMSF) and the root-mean-square deviation (RMSD) for each system were obtained by analyzing the trajectory using the VMD 1.8 program [37].

\subsection{The Binding Free Energies}

The binding free energies of the PP-3CL $\mathrm{C}^{\text {pro }}$ complexes were calculated by means of molecular mechanics-Poisson Boltzmann surface area (MM-PBSA) and molecular mechanics-generalized Born surface area (MM-GBSA) using the MMPBSA.py module of Amber Tools 21 [38]. The MD simulation over $200 \mathrm{~ns}$ provided several conformations sampled after equilibrium, using the last frames to lessen the computational cost. CPPTRAJ was used to obtain the snapshots [39]. The conformational changes were evaluated through quasi-harmonic entropy approximation [40]. The free energy of the binding interaction between inhibitors and $3 \mathrm{CL}^{\text {pro }}$ complexes can be obtained via the following equations:

$$
\begin{gathered}
\Delta \mathrm{G}=\Delta \mathrm{H}-\mathrm{T} \Delta \mathrm{S} \\
\Delta \mathrm{H}=\Delta \mathrm{G}_{\text {gas }}+\Delta \mathrm{G}_{\text {sol }} \\
\Delta \mathrm{G}_{\text {gas }}=\mathrm{E}_{\mathrm{vdw}}+\mathrm{E}_{\text {elec }} \\
\Delta \mathrm{G}_{\mathrm{sol}}=\mathrm{E}_{\mathrm{pb} / \mathrm{gb}}+\mathrm{E}_{\mathrm{np}}
\end{gathered}
$$

where $\Delta \mathrm{H}$ represents enthalpy change, $\mathrm{T} \Delta \mathrm{S}$ represents the entropic contribution, $\mathrm{E}_{\mathrm{vdw}}$ represents the van der Waals interaction energy, $\mathrm{E}_{\text {ele }}$ represents the electrostatic interaction energy, $\Delta \mathrm{G}_{\mathrm{sol}}$ represents the polar solvation energy, and $\mathrm{E}_{\mathrm{np}}$ represents the nonpolar solvation energy.

\section{Results and Discussion}

The results of the molecular docking are tabulated in Tables S1-S4. Figure 1 shows the catalytic dyad and the active sites of 3CL ${ }^{\text {pro }}$. The crucial residues HIS 41, GLY 143, SER 144, and CYS 145 forming the S1' site are shown in black. Then, PHE 140, LUE 141, ASN 142, HIS 163, GLU 166 (magenta), and the N-terminal amino acid residues (blue) are involved in the formation of the S1 subsite of the substrate-binding pocket. The MET 49, TYR 54, HIS 164, ASP 187, and ARG R188 residues form the S2 site (green). MET 165, LEU 167, GLN 189, THR 190, and GLN 192 comprise the S4 site (cyan). The SER 284, ALA 285, and LEU 286 residues (yellow) are a result of genetic mutation leading to an increase in the SASR-CoV-2 $3 \mathrm{CL}^{\text {pro }}$ activity of 3.6 fold over that of the $3 \mathrm{CL}^{\text {pro }}$ predecessor of SARS-CoV $[12,41]$. 


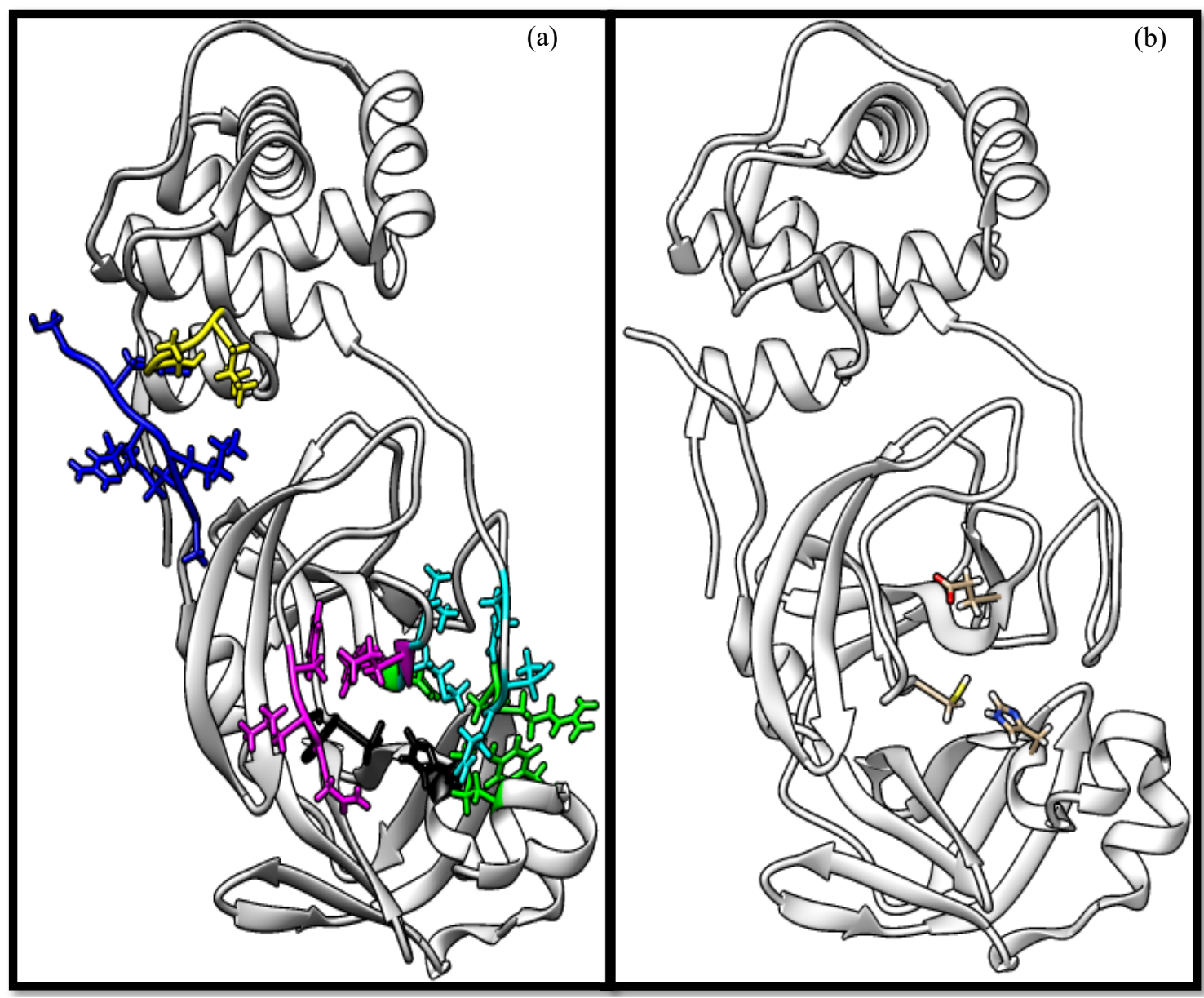

Figure 1. The crystal structure of chymotrypsin-like protease of SARS-CoV-2 (PDB ID: 6Y2E) and its active residues. (a) Color indicates the residues involved in the formation of the S1 site (shown in magenta), S1 site from the other promotor (blue), S2 site (green), S4 site (cyan), and S1' site (black), in addition to SER 284, ALA 285, and LEU 286 (yellow). (b) Only the catalytic dyad and GLU 166 residues.

\subsection{Molecular Docking}

The docked complexes of the top 11 candidates are depicted in Figure 2. Their binding affinities to the active sites of $3 \mathrm{CL}^{\text {pro }}$ are shown in Table 2. The 3PPs showed significant interactions with the residues LEU 286, SER 284, and ALA 285, and a relatively lower interaction ratio to the catalytic dyad, in contrast to the other groups. Of the 3PPs, alogliptin and flavin mononucleotide were found to have the highest binding percentage with the catalytic dyad and to form hydrogen bonds with the S1 and S'1 sites. These were followed by riboflavin and sofosbuvir with an advantage in binding to the LEU 286 residue (Table S1). Flavin adenine dinucleotide showed excellent binding affinity to LEU 286 but not with the catalytic dyad. Zidovudine and gemcitabine demonstrated similar activity to alogliptin and flavin mononucleotide (Tables S2 and S3).

Among the 2bPPs, anti-hepatitis B infection telbivudine, anti-orotic aciduria uridine triacetate, and anticancer tipiracil were found to have the highest binding to $3 \mathrm{CL}^{\text {pro }}$ active sites, followed by antimetabolite floxuridine, anti-herpesvirus trifluridine, and anti-HIV stavudine. Here, it is worth noting the importance of the molecular structure, as this set differed from the previous one by its increased ability to bind to the $3 \mathrm{CL}^{\text {pro }}$ catalytic dyad. The 2aPPs showed similar activity to that of the 2bPPs. Anticancer cytarabine, antiglaucoma citicoline, and anti-HIV drugs lamivudine and zalcitabine showed promising inhibitory activity (Table S3). 
Finally, but very importantly, of the 1PPs, the chemotherapy drug uracil mustard showed binding to the catalytic dyad with all of its simulated conformations, followed by anti-cytomegalovirus cidofovir (Table S4).

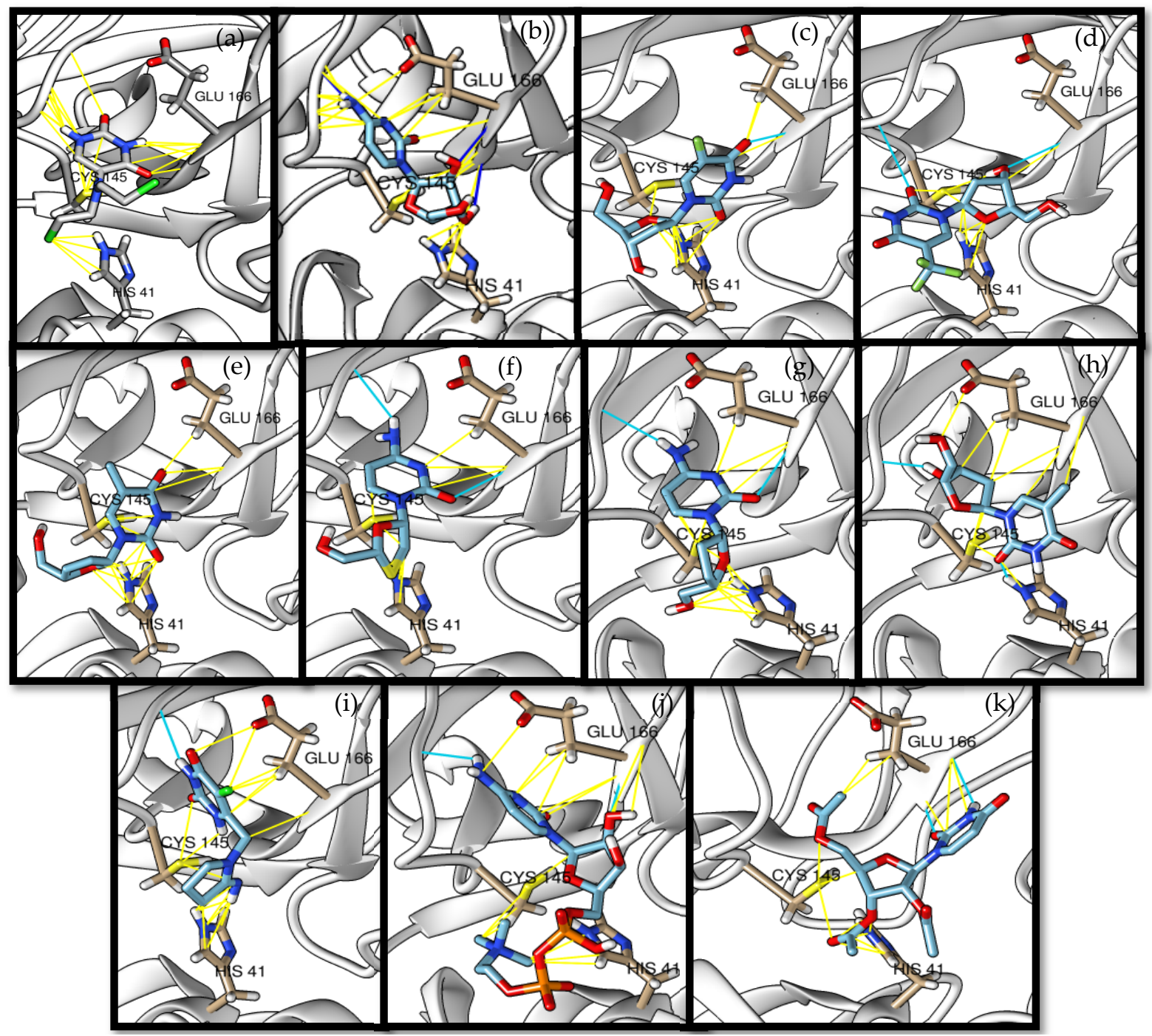

Figure 2. The PPs docked with 3CL pro, focusing on contacts with HIS 41, CYS 145, and GLU 166. (a) uracil mustard, (b) cytarabine, (c) floxuridine, (d) trifluridine, (e) stavudine, (f) lamivudine, (g) zalcitabine, (h) telbivudine, (i) tipiracil, (j) citicoline, (k) uridine triacetate. The hydrocarbon skeleton is shown in cyan, nitrogen atoms are blue, and oxygens are red. Hydrogen bonds are represented by blue lines; van der Waals forces are represented in yellow. 
Table 2. The binding affinities of the potential pyrimidonic pharmaceuticals with 3-chymotrypsin-like protease $\left(3 \mathrm{CL}^{\text {pro }}\right)$.

\begin{tabular}{|c|c|c|c|c|c|}
\hline $\begin{array}{l}\text { Pharmaceutical } \\
\text { Name }\end{array}$ & $\begin{array}{c}\text { Binding } \\
\text { Percentage }\end{array}$ & $\begin{array}{l}\text { Score } \pm \text { SD } \\
(\text { kcal } / \mathrm{mol})\end{array}$ & RMSD & $\begin{array}{l}\text { Hydrogen Bond (Number of } \\
\text { Bonds/Number of Conformations) }\end{array}$ & $\begin{array}{l}\text { Van Der Waals (Distance) (Number of Bonds/Number of } \\
\text { Conformations) }\end{array}$ \\
\hline Uracil mustard & $\begin{array}{l}\text { a. } 100 * \\
\text { c. } 89 \\
\text { d. } 100 \\
\text { e. } 100 \\
\text { All. } 100\end{array}$ & $\begin{array}{l}\text { a. }-4.6 \pm 0.14 \\
\text { c. }-4.6 \pm 0.14 \\
\text { d. }-4.6 \pm 0.14 \\
\text { e. }-4.6 \pm 0.14\end{array}$ & $\begin{array}{l}\text { a. } 0.00-7.13 \\
\text { c. } 0.00-7.13 \\
\text { d. } 0.00-7.13 \\
\text { e. } 0.00-7.13\end{array}$ & $\begin{array}{c}\text { a. HIS } 163 \text { (5/5), GLU } 166 \text { (3/3), LEU } 141 \\
(3 / 3), \text { ASN } 142(1 / 1)\end{array}$ & $\begin{array}{l}\text { a. HIS } 163(31 / 5) \text {, GLU } 166(40 / 8) \text {, LEU } 141(19 / 5), \text { ASN } 142 \\
\text { (50/7), PHE } 140(8 / 5) \\
\text { c. MET } 49(52 / 7), \text { HIS } 164(12 / 4) \\
\text { d. GLN } 189(32 / 9) \text {, MET } 165(28 / 8) \\
\text { e. HIS } 41 \text { (53/9), SER } 144(10 / 5), \text { GLY } 143(4 / 1), \text { CYS } 145(28 / 9)\end{array}$ \\
\hline Cytarabine & $\begin{array}{l}\text { a. } 67 \\
\text { c. } 67 \\
\text { d. } 67 \\
\text { e. } 67 \\
\text { All. } 67\end{array}$ & $\begin{array}{l}\text { a. }-5.4 \pm 0.26 \\
\text { c. }-5.4 \pm 0.26 \\
\text { d. }-5.4 \pm 0.26 \\
\text { e. }-5.4 \pm 0.26\end{array}$ & $\begin{array}{l}\text { a. } 0.00-6.07 \\
\text { c. } 0.00-6.07 \\
\text { d. } 0.00-6.07 \\
\text { e. } 0.00-6.07\end{array}$ & $\begin{array}{l}\text { a. GLU } 166(3 / 3), \text { LEU } 141(2 / 2), \text { HIS } \\
163(1 / 1), \text { PHE } 140(2 / 2), \text { ASN } 142(1 / 1) \\
\text { c. HIS } 164(2 / 2) \\
\text { d. GLN } 189(1 / 1) \\
\text { e. GLY } 143(1 / 1)\end{array}$ & $\begin{array}{l}\text { a. GLU } 166(44 / 5), \text { LEU } 141(18 / 3) \text {, HIS } 163(18 / 4) \text {, PHE } 140(19 / 3) \text {, } \\
\text { ASN } 142(27 / 6) \\
\text { c. HIS } 164(10 / 4) \text {, MET } 49(20 / 6) \\
\text { d. MET } 165(28 / 4) \text {, GLN } 189(4 / 2) \\
\text { e. GLY } 143(11 / 2) \text {, SER } 144(8 / 2) \text {, CYS } 145(15 / 6), \text { HIS } 41(21 / 5)\end{array}$ \\
\hline Floxuridine & $\begin{array}{l}\text { a. } 44 \\
\text { c. } 44 \\
\text { d. } 44 \\
\text { e. } 44 \\
\text { All. } 56 \\
\end{array}$ & $\begin{array}{l}\text { a. } 5.5 \pm 0.13 \\
\text { c. } 5.6 \pm 0.25 \\
\text { d. } 5.6 \pm 0.25 \\
\text { e. } 5.6 \pm 0.19\end{array}$ & $\begin{array}{l}\text { a. } 3.24-8.28 \\
\text { c. } 0.00-8.28 \\
\text { d. } 0.00-8.28 \\
\text { e. } 0.00-8.28\end{array}$ & $\begin{array}{l}\text { a. ASN } 142(1 / 1), \text { HIS } 163(2 / 2), \text { GLU } \\
166(2 / 2), \text { PHE } 140(1 / 1), \text { LEU } 141(1 / 1) \\
\text { e. HIS } 41(1 / 1)\end{array}$ & $\begin{array}{l}\text { a. GLU } 166(23 / 4), \text { LEU } 141 \text { (6/2), PHE } 140(8 / 2), \text { HIS } 163(5 / 2), \\
\text { ASN 142 (19/4) } \\
\text { c. MET } 49(9 / 2), \text { HIS } 164(3 / 3) \\
\text { d. MET } 165(5 / 3) \text {, GLN } 189(4 / 1) \\
\text { e. GLY } 143(6 / 1) \text {, SER } 144(4 / 1), \text { CYS } 145(5 / 3), \text { HIS } 41(16 / 3)\end{array}$ \\
\hline Trifluridine & $\begin{array}{c}\text { a. } 44 \\
\text { c. } 44 \\
\text { d. } 44 \\
\text { e. } 44 \\
\text { f. } 11 \\
\text { All. } 56\end{array}$ & $\begin{array}{l}\text { a. }-6.03 \pm 0.17 \\
\text { c. }-6.03 \pm 0.17 \\
\text { d. }-6.03 \pm 0.17 \\
\text { e. }-6.03 \pm 0.17 \\
\quad \text { f. }-5.7\end{array}$ & $\begin{array}{l}\text { a. } 0.00-5.58 \\
\text { c. } 0.00-5.58 \\
\text { d. } 0.00-5.58 \\
\text { e. } 0.00-5.58 \\
\text { f. } 28.34-30.21\end{array}$ & $\begin{array}{l}\text { a. GLU } 166(2 / 2), \text { ASN } 142(1 / 1) \text {. } \\
\text { c. HIS } 164(2 / 2) \\
\text { e. GLY } 143(1 / 1)\end{array}$ & $\begin{array}{c}\text { a. GLU } 166(18 / 4), \text { ASN } 142(15 / 3) \text {, HIS } 163(2 / 1) \text {, LEU } 141(2 / 1) \\
\text { b. } \\
\text { c. HIS } 164(10 / 3), \text { MET } 49(17 / 4) \\
\text { d. MET } 165(19 / 4), \text { GLN } 189(1 / 1) \\
\text { e. CYS } 145(13 / 4), \text { GLY } 143(10 / 2), \text { HIS } 41(10 / 4) \\
\text { f. SER } 284(9 / 1)\end{array}$ \\
\hline Stavudine & $\begin{array}{l}\text { a. } 44 \\
\text { c. } 56 \\
\text { d. } 44 \\
\text { e. } 56 \\
\text { All. } 56\end{array}$ & $\begin{array}{l}\text { a. }-5.6 \pm 0.28 \\
\text { c. }-5.6 \pm 0.28 \\
\text { d. }-5.6 \pm 0.28 \\
\text { e. }-5.6 \pm 0.28\end{array}$ & $\begin{array}{l}\text { a. } 27.12-32.34 \\
\text { c. } 27.12-35.03 \\
\text { d. } 27.12-32.34 \\
\text { e. } 27.12-35.03\end{array}$ & a. GLU $166(1 / 1)$ & $\begin{array}{l}\text { a. ASN } 142(13 / 3) \text {, GLU } 166(14 / 4) \text {, HIS } 163(2 / 1) \text {, LEU } 141 \text { (1/1) } \\
\text { c. HIS } 164(6 / 3) \text {, MET } 49(20 / 5) \\
\text { d. MET } 165(12 / 4) \text {, GLN } 189(1 / 1) \\
\text { e. HIS } 41 \text { (39/5), GLY } 143(9 / 2) \text {, CYS } 145(7 / 3)\end{array}$ \\
\hline Lamivudine & $\begin{array}{l}\text { a. } 56 \\
\text { c. } 44 \\
\text { d. } 44 \\
\text { e. } 56 \\
\text { f. } 11 \\
\text { All. } 67\end{array}$ & $\begin{array}{l}\text { a. }-5.4 \pm 0.24 \\
\text { c. }-5.4 \pm 0.28 \\
\text { d. }-5.4 \pm 0.25 \\
\text { e. }-5.4 \pm 0.24 \\
\quad \text { f. }-5.2\end{array}$ & $\begin{array}{l}\text { a. } 0.00-4.77 \\
\text { c. } 0.00-3.38 \\
\text { d. } 0.00-4.77 \\
\text { e. } 0.00-4.77 \\
\text { f. } 26.28-28.62\end{array}$ & $\begin{array}{c}\text { a. HIS } 163(3 / 3), \text { ASN } 142(1 / 1), \text { PHE } \\
140(3 / 3), \text { LEU } 141(2 / 2), \text { GLU } 166(2 / 2) \\
\text { d. GLN } 189(1 / 1) \\
\text { e. SER } 144(2 / 2)\end{array}$ & $\begin{array}{c}\text { a. HIS } 163(26 / 5) \text {, ASN } 142(19 / 2), \text { PHE } 140(31 / 5) \text {, LEU } 141(15 / 5), \\
\text { GLU } 166(46 / 5) \\
\text { c. MET } 49(12 / 4), \text { HIS } 164(1 / 1) \\
\text { d. GLN } 189(12 / 2), \text { MET } 165(10 / 4), \text { LEU } 167(1 / 1) \\
\text { e. SER } 144(18 / 4), \text { HIS } 41(5 / 2), \text { CYS } 145(7 / 3) \\
\text { f. LEU } 286(1 / 1)\end{array}$ \\
\hline
\end{tabular}


Table 2. Cont.

\begin{tabular}{|c|c|c|c|c|c|}
\hline $\begin{array}{l}\text { Pharmaceutical } \\
\text { Name }\end{array}$ & $\begin{array}{c}\text { Binding } \\
\text { Percentage }\end{array}$ & $\begin{array}{l}\text { Score } \pm \text { SD } \\
(\text { kcal } / \text { mol })\end{array}$ & RMSD & $\begin{array}{l}\text { Hydrogen Bond (Number of } \\
\text { Bonds/Number of Conformations) }\end{array}$ & $\begin{array}{l}\text { Van Der Waals (Distance) (Number of Bonds/Number of } \\
\text { Conformations) }\end{array}$ \\
\hline Zalcitabine & $\begin{array}{l}\text { a. } 44 \\
\text { b. } 11 \\
\text { c. } 33 \\
\text { d. } 44 \\
\text { e. } 44 \\
\text { f. } 22 \\
\text { All. } 67\end{array}$ & $\begin{array}{l}\text { a. }-5.5 \pm 0.29 \\
\quad \text { b. }-5.1 \\
\text { c. }-5.4 \pm 0.35 \\
\text { d. }-5.5 \pm 0.29 \\
\text { e. }-5.5 \pm 0.29 \\
\text { f. }-5.1 \pm 0.00\end{array}$ & $\begin{array}{l}\text { a. } 0.00-6.39 \\
\text { b. } 28.02-29.48 \\
\text { c. } 0.00-6.39 \\
\text { d. } 0.00-6.39 \\
\text { e. } 0.00-6.39 \\
\text { f. } 28.02-31.88\end{array}$ & $\begin{array}{c}\text { a. PHE } 140(3 / 3), \text { LEU } 141(1 / 1), \text { GLU } \\
166(3 / 3), \text { ASN } 142(1 / 1) \\
\text { c. GLN } 189(1 / 1)\end{array}$ & $\begin{array}{l}\text { a. HIS } 163 \text { (15/3), PHE } 140(16 / 3) \text {, LEU } 141 \text { (9/3), GLU } 166(25 / 4) \text {, } \\
\text { ASN } 142(11 / 3) \\
\text { b. LYS } 5 \text { (6/1), ARG } 4(12 / 1) \text {, PHE } 3(4 / 1) \\
\text { c. MET } 49(16 / 2), \text { HIS } 164(5 / 3) \\
\text { d. GLN } 189(6 / 2) \text {, MET } 165(15 / 4) \\
\text { e. SER } 144 \text { (7/2), HIS 41 (13/2), CYS } 145(6 / 3) \\
\text { f. SER 284 (4/1), LEU 286 (4/1) }\end{array}$ \\
\hline Telbivudine & $\begin{array}{l}\text { a. } 56 \\
\text { c. } 44 \\
\text { d. } 44 \\
\text { e. } 56 \\
\text { f. } 11 \\
\text { All. } 67\end{array}$ & $\begin{array}{l}\text { a. }-5.6 \pm 0.38 \\
\text { c. }-5.7 \pm 0.42 \\
\text { d. }-5.7 \pm 0.42 \\
\text { e. }-5.6 \pm 0.38 \\
\quad \text { f. }-5.3\end{array}$ & $\begin{array}{l}\text { a. } 0.00-7.50 \\
\text { c. } 0.00-7.50 \\
\text { d. } 0.00-7.50 \\
\text { e. } 0.00-7.50 \\
\text { f. } 22.85-24.17\end{array}$ & $\begin{array}{l}\text { a. ASN } 142(2 / 1), \text { HIS } 163(3 / 3), \text { GLU } \\
166(1 / 1), \text { PHE } 140(2 / 2), \text { LEU } 141(1 / 1) \\
\text { c. HIS } 164(1 / 1) \\
\text { d. GLN } 189(1 / 1) \\
\text { e. HIS } 41(1 / 1)\end{array}$ & $\begin{array}{r}\text { a. GLU } 166(36 / 4), \text { HIS } 163(11 / 3), \text { PHE } 140(11 / 3), \text { ASN } 142 \\
\text { (27/5), LEU } 141(9 / 3) \\
\text { c. HIS } 164(6 / 3), \text { MET } 49(27 / 3) \\
\text { d. GLN } 189(10 / 3) \text {, MET } 165(14 / 4), \\
\text { e. CYS } 145(11 / 4) \text {, SER } 144(8 / 2) \text {, GLY } 143(3 / 1), \text { HIS } 41(12 / 5) \\
\text { f. LEU 286 (5/1) }\end{array}$ \\
\hline Tipiracil & $\begin{array}{l}\text { a. } 56 \\
\text { b. } 11 \\
\text { c. } 44 \\
\text { d. } 44 \\
\text { e. } 44 \\
\text { f. } 11 \\
\text { All. } 67\end{array}$ & $\begin{array}{l}\text { a. }-5.8 \pm 0.16 \\
\quad \text { b. }-5.7 \\
\text { c. }-5.9 \pm 0.17 \\
\text { d. }-5.8 \pm 0.08 \\
\text { e. }-5.9 \pm 0.17 \\
\quad \text { f. }-5.7\end{array}$ & $\begin{array}{l}\text { a. } 26.28-29.69 \\
\text { b. } 19.12-20.08 \\
\text { c. } 26.28-29.69 \\
\text { d. } 26.67-29.69 \\
\text { e. } 26.28-29.69 \\
\text { f. } 19.12-20.08\end{array}$ & $\begin{array}{l}\text { a. HIS } 163(2 / 2), \text { GLU } 166(2 / 1) \text {, PHE } \\
140(2 / 2), \operatorname{ASN} 142(1 / 1) \\
\text { b. LYS } 5(1 / 1) \\
\text { c. HIS } 164(1 / 1) \\
\text { e. GLY } 143(1 / 1)\end{array}$ & $\begin{array}{l}\text { a. HIS } 163(14 / 3) \text {, GLU } 166(40 / 4), \text { PHE } 140(8 / 3), \text { ASN } 142(22 / 4) \text {, } \\
\text { LEU } 141(10 / 3) \\
\text { b. PHE } 3(6 / 1) \text {, LYS } 5(9 / 1), \text { ARG } 4(5 / 1) \\
\text { c. HIS } 164(8 / 4), \text { MET } 49(22 / 3) \\
\text { d. MET } 165(9 / 3) \text {, GLN } 189(13 / 3) \\
\text { e. GLY } 143(7 / 2) \text {, HIS } 41(26 / 4) \text {, SER } 144(2 / 1), \text { CYS } 145(13 / 4) \\
\text { f. SER } 284(7 / 1) \text {, LEU } 286(2 / 1)\end{array}$ \\
\hline Citicoline & $\begin{array}{l}\text { a. } 56^{*} \\
\text { c. } 56 \\
\text { d. } 56 \\
\text { e. } 56 \\
\text { All. } 56 \\
\end{array}$ & $\begin{array}{l}\text { a. }-7.0 \pm 0.19 \\
\text { c. }-7.0 \pm 0.19 \\
\text { d. }-7.0 \pm 0.19 \\
\text { e. }-7.0 \pm 0.19\end{array}$ & $\begin{array}{l}\text { a. } 0.00-8.01 \\
\text { c. } 0.00-8.01 \\
\text { d. } 0.00-8.01 \\
\text { e. } 0.00-8.01\end{array}$ & $\begin{array}{c}\text { a. PHE } 140(2 / 2), \text { GLU } 166(4 / 4), \text { HIS } \\
163(3 / 3), \text { ASN } 142(2 / 1), \text { LEU } 141(1 / 1) \\
\text { e. SER } 144(1 / 1)\end{array}$ & $\begin{array}{c}\text { a. PHE } 140(24 / 5) \text {, GLU } 166(57 / 5), \text { HIS } 163(21 / 5), \text { ASN } 142 \\
(34 / 5), \text { LEU } 141(21 / 5) \\
\text { c. MET } 49(23 / 5), \text { HIS } 164(1 / 1) \\
\text { d. MET } 165(29 / 5) \text {, GLN } 189(6 / 3) \\
\text { e. SER } 144(12 / 3) \text {, GLY } 143(4 / 1) \text {, CYS } 145(9 / 5), \text { HIS } 41(21 / 5)\end{array}$ \\
\hline $\begin{array}{l}\text { Uridine } \\
\text { triacetate }\end{array}$ & $\begin{array}{l}\text { a. } 56 \\
\text { c. } 56 \\
\text { d. } 56 \\
\text { e. } 56 \\
\text { f. } 11 \\
\text { All. } 67\end{array}$ & $\begin{array}{l}\text { a. }-6.2 \pm 0.26 \\
\text { c. }-6.2 \pm 0.26 \\
\text { d. }-6.2 \pm 0.26 \\
\text { e. }-6.2 \pm 0.26 \\
\quad \text { f. }-6.4\end{array}$ & $\begin{array}{l}\text { a. } 0.00-6.79 \\
\text { c. } 0.00-6.79 \\
\text { d. } 0.00-6.79 \\
\text { e. } 0.00-6.79 \\
\text { f. } 24.97-28.01\end{array}$ & $\begin{array}{c}\text { a. HIS } 163(2 / 2), \text { GLU } 166(3 / 2) \\
\text { e. HIS } 41(1 / 1)\end{array}$ & $\begin{array}{l}\text { a. HIS } 163(5 / 2), \text { GLU } 166(31 / 5), \text { ASN } 142(30 / 5), \text { PHE } 140(4 / 2) \text {, } \\
\text { LEU } 141 \text { (6/2) } \\
\text { c. MET } 49(18 / 5), \text { HIS } 164(5 / 2) \\
\text { d. GLN } 189(15 / 4), \text { MET } 165(18 / 4) \\
\text { e. CYS } 145(16 / 5), \text { HIS } 41(35 / 5), \text { SER } 144(4 / 2), \text { GLY } 143(2 / 1) \\
\text { f. LEU } 286(1 / 1)\end{array}$ \\
\hline
\end{tabular}

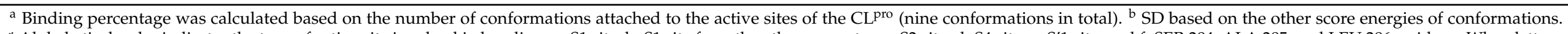

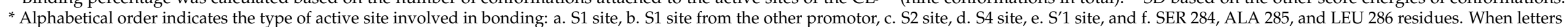
are missing, this means no interactions were observed at that site. 


\subsection{Molecular Dynamics Simulations}

MD was performed on the hole complexes of the top 11 PPs candidates. Based on the conformer score energy from docking, the complex with the conformer with the lowest value and interacting with the $3 \mathrm{CL}^{\text {pro }}$ active site was selected. The RMSDs were computed along the trajectories using the initial structure as a reference. Figure 3 shows that the binding of PPs significantly affected the equilibration states of $3 C^{2}{ }^{\text {pro }}$, as the majority of the tested systems reached their equilibrium at around $100 \mathrm{~ns}$. The PP-3CLpro complexes revealed relatively lower average values for the RMSDs, between 0.41 and $0.52 \AA$, throughout the simulation, clarifying their good behavior in forming stable complexes. Moreover, the fluctuations in the $3 \mathrm{CL}^{\text {pro }}$ backbone residues were analyzed by means of the RMSF (Figure 4). The 3CL pro /PPs exhibited lower fluctuations, particularly at the active site. The fluctuations at the catalytic dyad and GLU 166 were minor, demonstrating the loss of flexibility at these regions upon binding to the PPs. Table 3 shows the superior stability of the PP-3CL $\mathrm{CL}^{\text {pro }}$ complexes formed throughout the production runs; these results support the use of these PPs as $3 \mathrm{CL}^{\mathrm{pro}}$ inhibitors.

Table 3. The binding interactions of the potential pyrimidonic pharmaceuticals/3-chymotrypsin-like protease 3CL pro complexes at different times throughout the production runs.

$\begin{gathered}\text { Pharmaceutical } \\ \text { Name }\end{gathered}$
Uracil mustard
Floxuridine


Table 3. Cont.

\section{Pharmaceutical}

Name

$100 \mathrm{~ns}$

150 ns

200 ns

Trifluridine
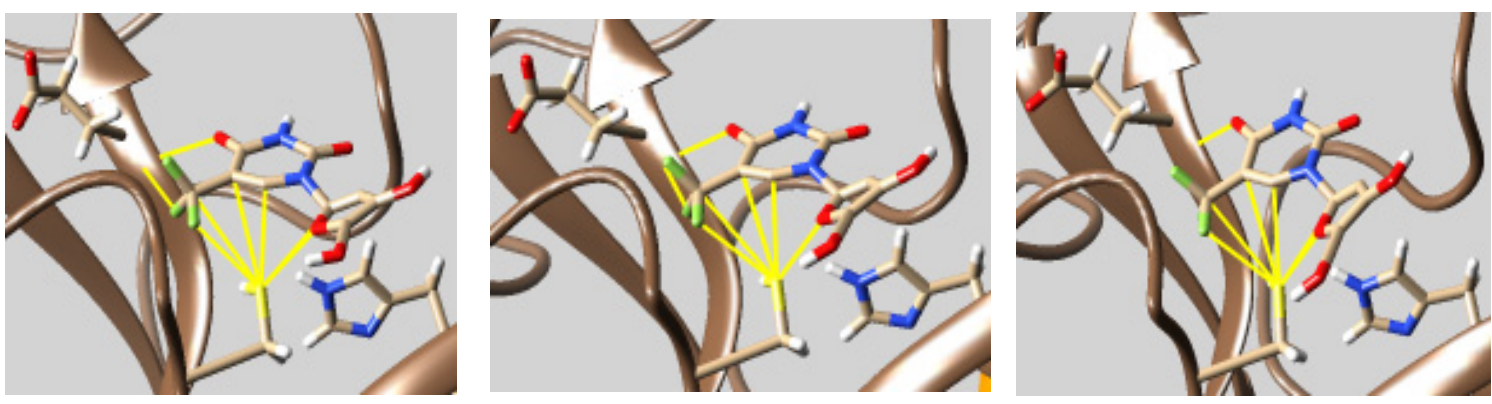

Stavudine
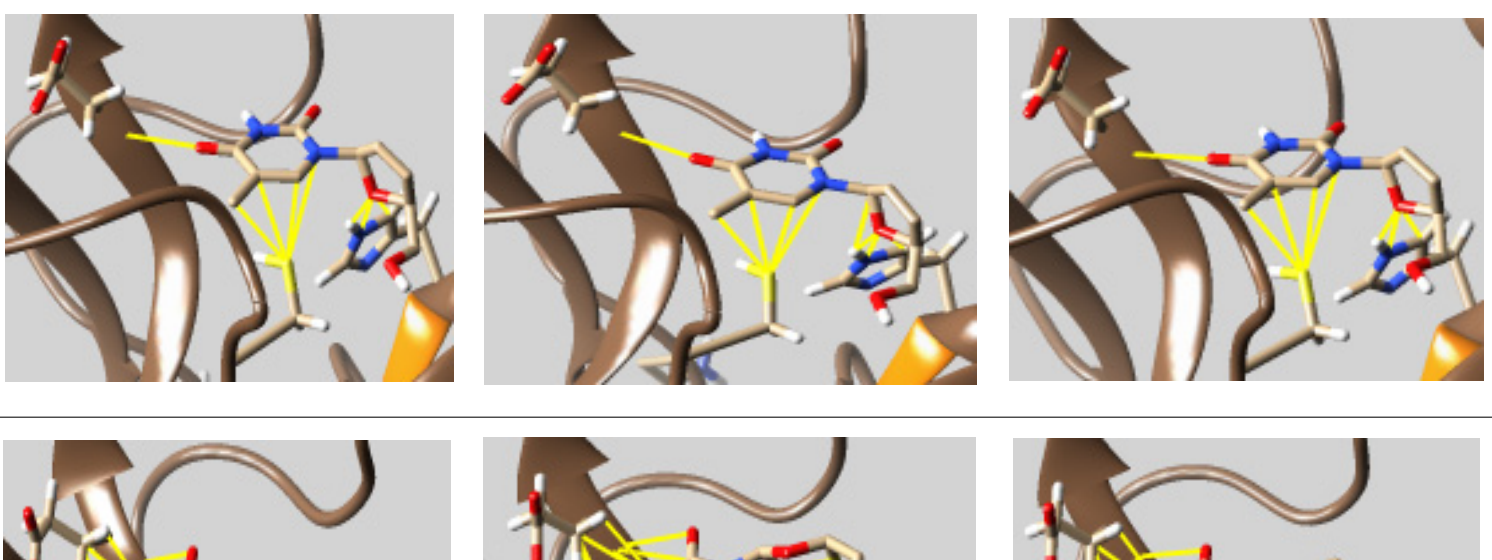

Lamivudine
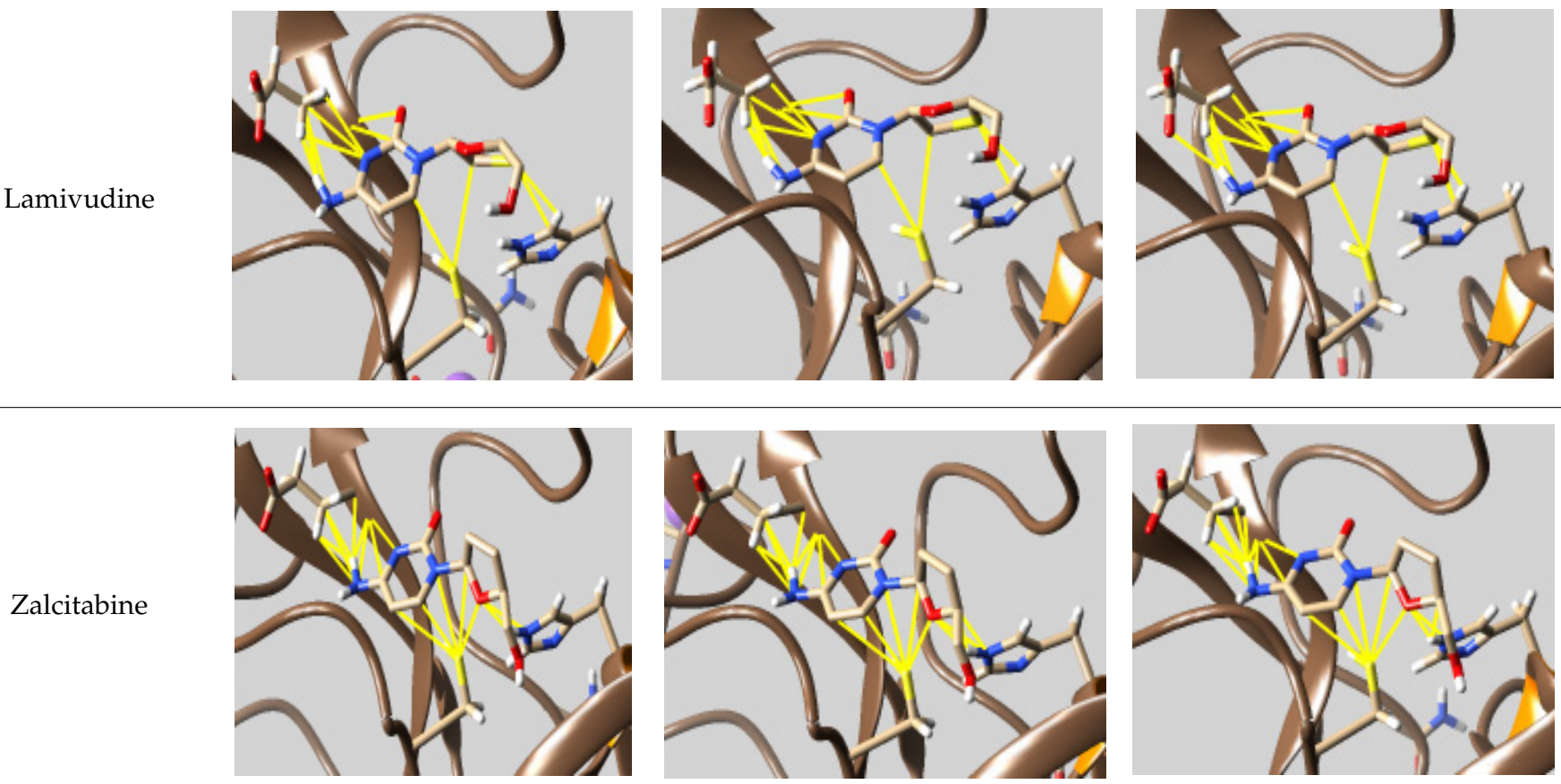

Telbivudine
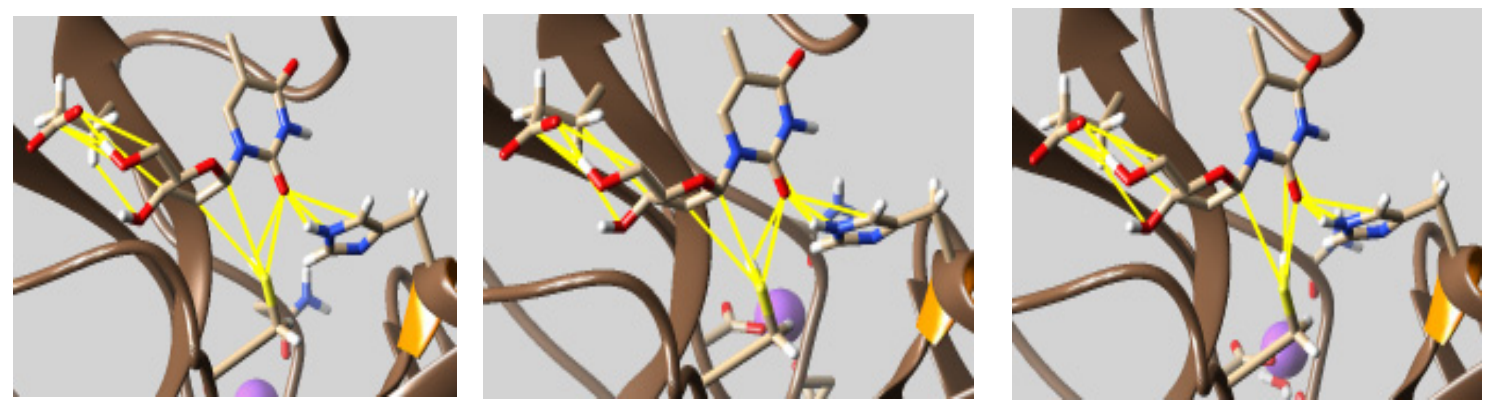
Table 3. Cont

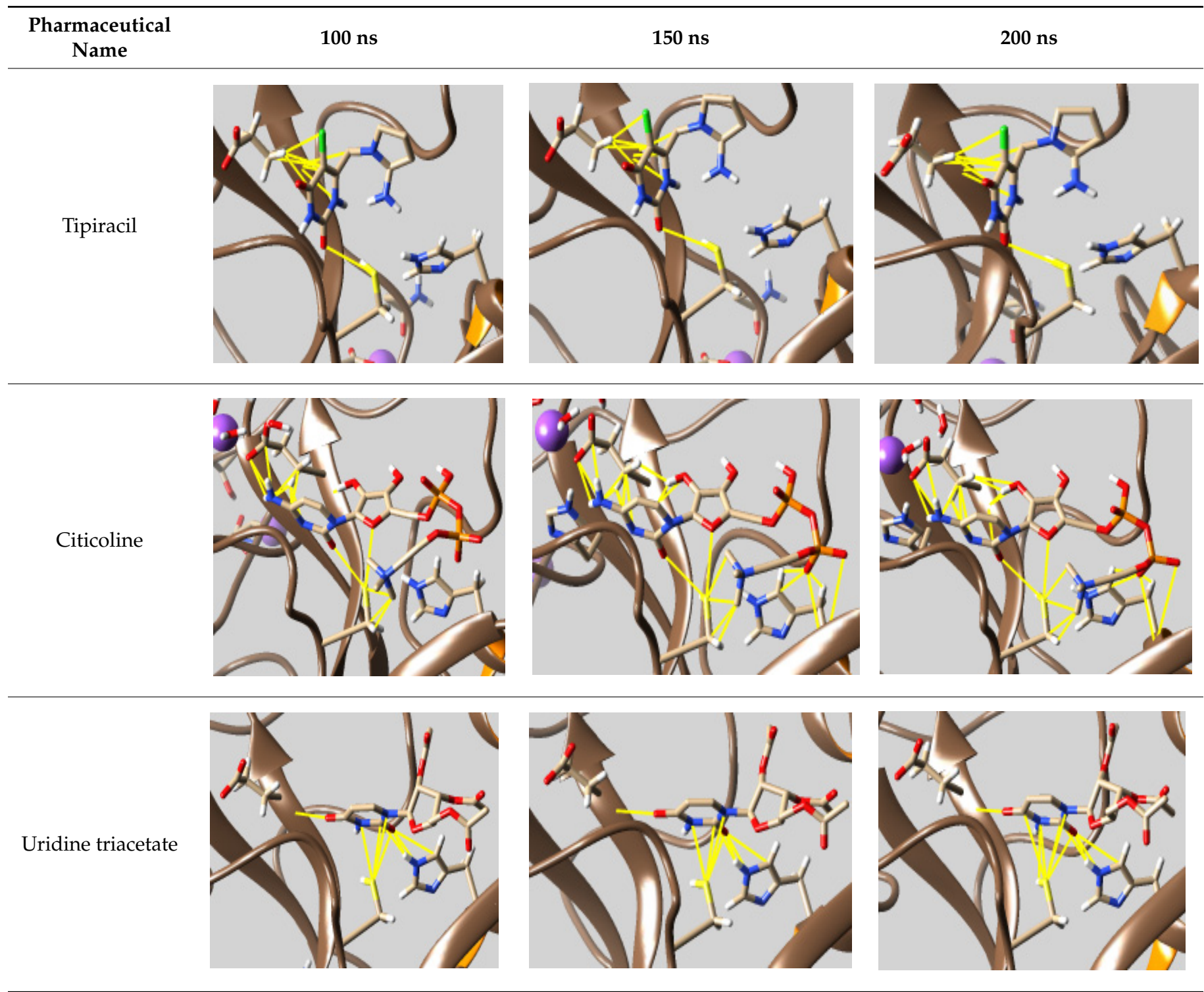

$\bullet$ Uracil mustard $\quad$ Cytarabine $\triangle$ Floxuridine $\times$ Trifluridine

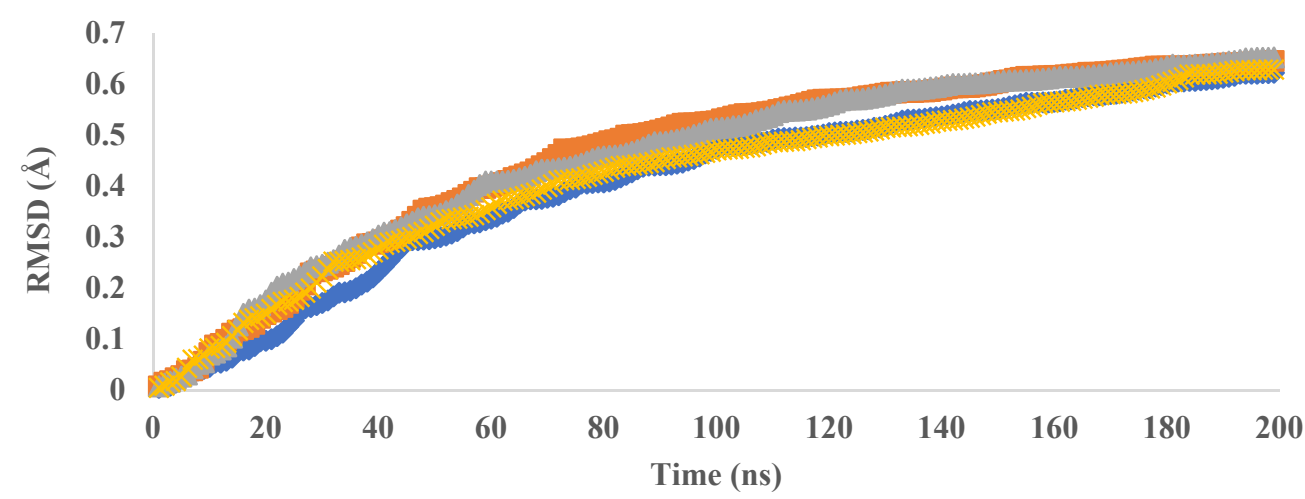

Figure 3. Cont. 


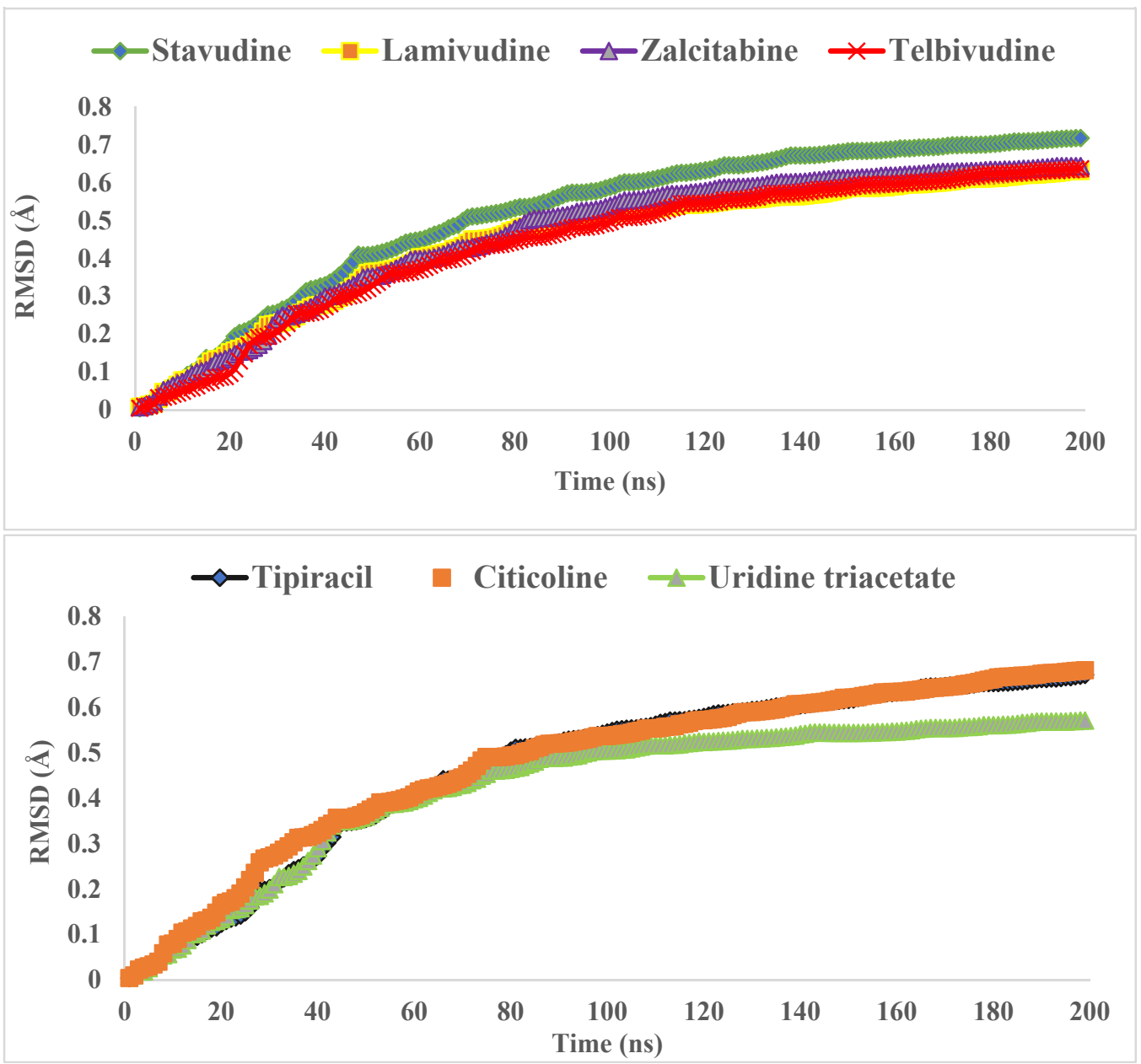

Figure 3. The RMSD values of the simulated PP-3CL ${ }^{\text {pro }}$ complexes throughout the 200 ns production runs.

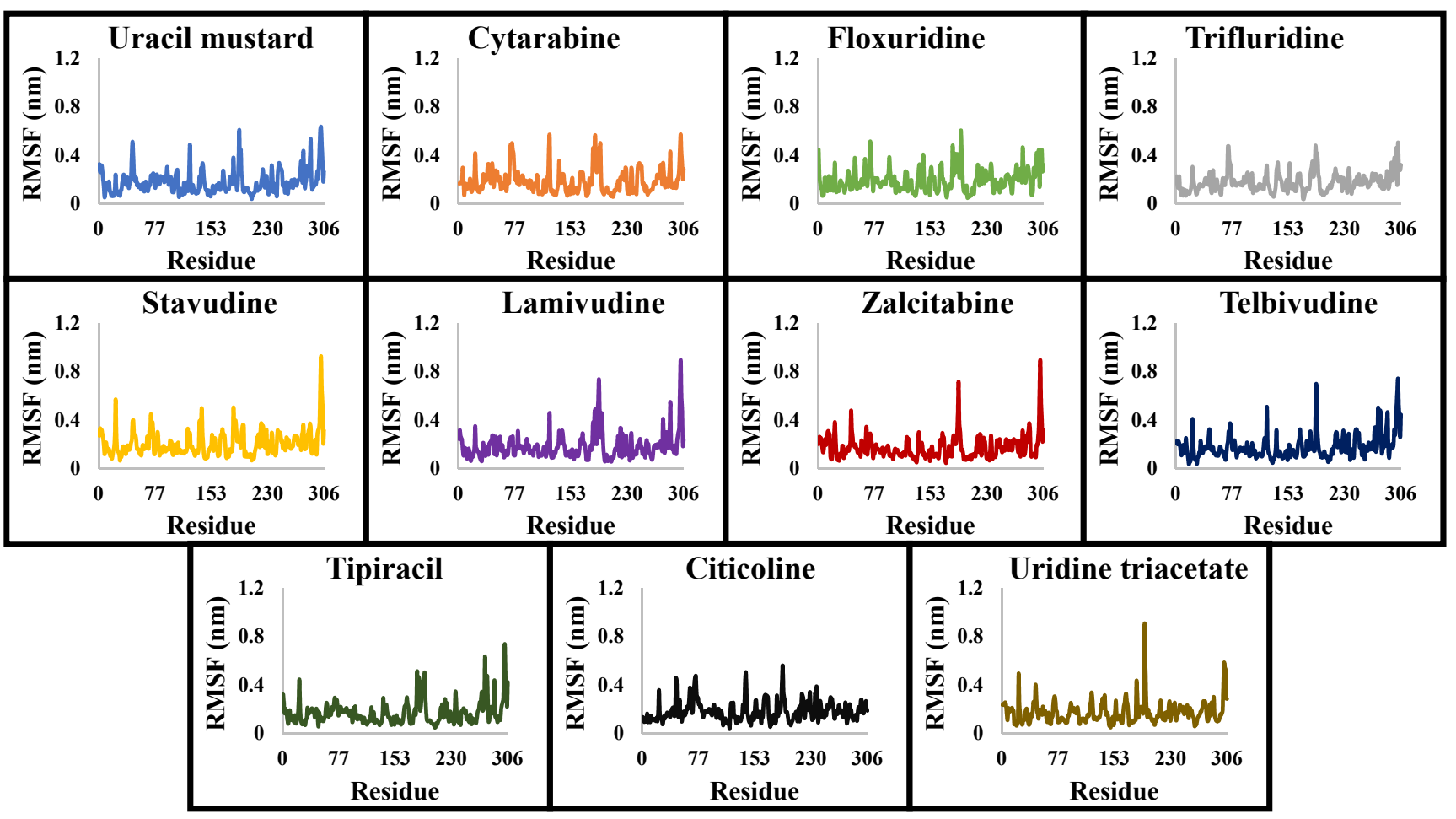

Figure 4. The RMSF values of the simulated PP-3CL ${ }^{\text {pro }}$ complexes throughout the 200 ns production runs. 


\subsection{The Binding Free Energies}

The data on the binding free energies of the PP-3CL ${ }^{\text {pro }}$ complexes are tabulated in Table 4. In both MM-GBSA and MM-PBSA, van der Waals and electrostatic interactions acted as driving forces for the PP ligands to bind to $3 \mathrm{CL}^{\text {pro }}$, contrasting the solvation energies. The MM-GBSA and MM-PBSA results suggest that the PPs have an excellent ability to inhibit $3 \mathrm{CL}^{\text {pro }}$. Of these PPs, citicoline revealed the most promising inhibitory activity, followed by uridine triacetate (Table 4).

Table 4. The MMPBSA and MMGBSA data for the binding of pyrimidone containing-pharmaceuticals to $3 C L^{\text {Pro }}$ of SARS-CoV-2.

\begin{tabular}{|c|c|c|c|c|c|c|c|c|}
\hline \multirow{2}{*}{$\begin{array}{c}\text { 3CL pro Complex } \\
\text { Type }\end{array}$} & \multirow{2}{*}{$-\mathrm{T} \Delta \mathrm{S}$} & \multirow{2}{*}{$E_{v d w}$} & \multicolumn{3}{|c|}{ MMGBSA } & \multicolumn{3}{|c|}{ MMPBSA } \\
\hline & & & $E_{\text {alac }}$ & $\mathrm{E}_{\mathrm{sol}}$ & $\Delta \mathrm{g}(\mathrm{kcal} / \mathrm{mol})$ & $E_{\text {alac }}$ & $\mathrm{E}_{\mathrm{sol}}$ & $\Delta \mathrm{g}(\mathrm{kcal} / \mathrm{mol})$ \\
\hline Uracil mustard & 22.58 & -23.76 & -16.08 & 20.46 & 3.20 & -0.80 & 0.94 & -1.05 \\
\hline Cytarabine & 22.39 & -19.25 & -33.50 & 28.39 & -1.96 & -1.67 & 1.09 & 2.55 \\
\hline Floxuridine & 22.42 & -24.54 & 0.00 & 3.70 & 1.58 & 0.00 & 0.39 & -1.73 \\
\hline Trifluridine & 22.96 & -29.48 & 0.00 & 4.20 & -2.32 & 0.00 & 0.41 & -6.11 \\
\hline Stavudine & 22.25 & -24.41 & -17.67 & 17.90 & -1.94 & -0.88 & 0.93 & -2.12 \\
\hline Lamivudine & 22.26 & -20.58 & -35.11 & 28.94 & -4.50 & -1.76 & 1.06 & 0.98 \\
\hline Zalcitabine & 22.12 & -24.34 & -30.10 & 26.50 & -5.81 & -1.50 & 1.19 & 2.53 \\
\hline Telbivudine & 22.48 & -25.29 & -46.88 & 42.53 & -7.16 & -2.34 & 1.61 & -3.54 \\
\hline Tipiracil & 22.54 & -28.79 & 0.00 & 5.07 & -1.18 & 0.00 & 0.44 & -5.81 \\
\hline Citicoline & 24.17 & -54.50 & 0.00 & 4.80 & -25.53 & 0.00 & 0.64 & -29.69 \\
\hline Uridine triacetate & 23.60 & -32.98 & -31.09 & 33.40 & -7.07 & -1.55 & 1.43 & -9.51 \\
\hline
\end{tabular}

Among the challenges of discovering 3CL pro inhibitors for COVID-19 treatment, these inhibitors must be highly bioavailable inside the cytosol [13]. The 2aPP and 2bPP structures contain a primidone heterocycle and ribose ring. These heterocycles increase their hydrophilicity and solubility in plasma. Thus, they can satisfy the requirement of bioavailability. Citicoline, the most promising inhibitor among the PPs investigated, has high hydrophilicity and good ADME properties [42,43]. The 2aPPs and 2bPPs also have an intermediate structure size among the PP groups. This sheds light on the importance of the size and general structural features of PPs acting as $3 \mathrm{CL}^{\text {pro }}$ inhibitors.

Recent reports suggest a general hypothesis that $3 \mathrm{CL}^{\text {pro }}$ inhibitors comprise electrophilic sites such as Michael acceptors [12]. That the pyrimidone ring is highly electrondeficient clarifies and confirms this hypothesis. The pyrimidone ring plays an essential role in PPs' inhibitory activity and has a high tendency to form hydrogen bonds, particularly with the GLU 166 residue. This may preclude the formation of the S1 pocket. Contacts between the pyrimidone ring and HIS 41 were observed in floxuridine, stavudine, and telbivudine. In all these cases, HIS 41 interacts with the oxygen of the pyrimidone group. Further, the electrophilic carbon, nitrogen, and oxygen in the pyrimidone ring were attracted to bind with the sulfur of the CYS 145 residue.

Interestingly, most PPs investigated were previously studied against SARS-CoV-2. For example, flavin mononucleotide and flavin adenine dinucleotide have been suggested as good $3 \mathrm{CL}^{\text {pro }}$ and RNA-dependent RNA polymerase inhibitors, respectively [22,44]. Riboflavin and sofosbuvir were shown to be suitable inhibitors of the spike protein S1 domain/ACE2 and RNA-dependent RNA polymerase [45-47]. Alogliptin was also suggested as a $3 \mathrm{CL}^{\text {pro }}$ inhibitor; however, the enzymatic assay demonstrated its inactivity against $3 \mathrm{CL}^{\text {pro }}$ [48]. Compelling clues have been found regarding the use of zidovudine and gemcitabine against spike protein/human ACE2 and in the inhibition SARS-CoV-2 in cell culture [49-53]. Gemcitabine and cidofovir were reported to inhibit SARS-CoV and SARS-CoV-2 proteins with $\mathrm{IC}_{50}$ values of $4.95 \mu \mathrm{M}$ and $36 \mu \mathrm{M}[54,55]$. Further, telbivudine, tipiracil, cytarabine, and citicoline were recommended as $3 \mathrm{CL}^{\text {pro }}$ inhibitors [56-61]. This transitory literature scanning confirms these pharmaceuticals' activity against $3 \mathrm{CL}^{\text {pro }}$ of SARS-CoV-2, as demonstrated in the present study. 
To conclude, the inhibitory effect on $3 C L^{\text {pro }}$ by PPs was investigated based on their ability to form hydrogen bonds and van der Waals interactions with the $3 \mathrm{CL}^{\text {pro }}$ active side through molecular docking, MD simulations, and the calculation of binding free energy. The overall analysis revealed 11 candidates from the initial set of 42 investigated PPs are promising $3 \mathrm{CL}^{\text {pro }}$ inhibitors. These include citicoline and uridine triacetate as the best choices, followed by telbivudine, trifluridine, lamivudine, cytarabine, stavudine, zalcitabine, tipiracil, floxuridine, and flavin mononucleotide. The interactions of PPs with the catalytic dyad and the active sides of $3 \mathrm{CL}^{\text {pro }}$ of SARS-CoV-2 were comprehensively and thoroughly investigated. The pyrimidone ring was found to play an essential role in the PPs' inhibitory activity.

Supplementary Materials: The following are available online, Table S1: The binding affinities of the pyrimidonic pharmaceuticals (group 3PPs) with 3-chymotrypsin-like protease (3CLpro), Table S2: The binding affinities of the pyrimidonic pharmaceuticals (group 2bPPs) with 3-chymotrypsin-like protease (3CLpro), Table S3: The binding affinities of the pyrimidonic pharmaceuticals (group $2 \mathrm{aPPs}$ ) with 3-chymotrypsin-like protease (3CLpro), Table S4: The binding affinity of the pyrimidone containing-pharmaceuticals (group 1PCPs) with 3-chymotrypsinlike protease (3CLpro).

Funding: This research and the APC were funded by the Deanship of Scientific Research, Imam Mohammad Ibn Saud Islamic University (IMSIU), Saudi Arabia, grant number [21-13-18-040].

Institutional Review Board Statement: Not applicable.

Informed Consent Statement: Not applicable.

Data Availability Statement: The data will be available upon request.

Conflicts of Interest: There is no conflict of interest to be reported.

Sample Availability: Not applicable.

\section{References}

1. Ledford, H.; Cyranoski, D.; Van Noorden, R. The UK has approved a COVID vaccine-here's what scientists now want to know. Nature 2020, 588, 205-206. [CrossRef]

2. Mahase, E. Covid-19: UK approves Moderna vaccine to be given as two doses 28 days apart. BMJ 2021, 372. [CrossRef] [PubMed]

3. Mahase, E. Covid-19: Moderna applies for US and EU approval as vaccine trial reports 94.1\% efficacy. BMJ 2020, 371. [CrossRef] [PubMed]

4. Lamb, Y.N. Remdesivir: First approval. Drugs 2020, 80, 1355-1363. [CrossRef]

5. Rubin, D.; Chan-Tack, K.; Farley, J.; Sherwat, A. FDA approval of remdesivir-A step in the right direction. N. Engl. J. Med. 2020, 383, 2598-2600. [CrossRef]

6. Rivero-Segura, N.A.; Gomez-Verjan, J.C. In Silico Screening of Natural Products Isolated from Mexican Herbal Medicines against COVID-19. Biomolecules 2021, 11, 216. [CrossRef] [PubMed]

7. World Health Organization. Coronavirus Disease 2019 (COVID-19): Situation Report, 94. 2020. Available online: https: / / apps.who.int/iris/handle/10665/331865 (accessed on 27 November 2021).

8. Zhou, P.; Yang, X.-L.; Wang, X.-G.; Hu, B.; Zhang, L.; Zhang, W.; Si, H.-R.; Zhu, Y.; Li, B.; Huang, C.-L. A pneumonia outbreak associated with a new coronavirus of probable bat origin. Nature 2020, 579, 270-273. [CrossRef]

9. World Health Organization. COVID-19 Weekly Epidemiological Update. 2020. Available online: https://www.who.int/ emergencies/diseases/novel-coronavirus-2019/situation-reports (accessed on 2 December 2021).

10. Ullrich, S.; Nitsche, C. The SARS-CoV-2 main protease as drug target. Bioorganic Med. Chem. Lett. 2020, 30, 127377. [CrossRef]

11. Zhang, L.; Lin, D.; Sun, X.; Curth, U.; Drosten, C.; Sauerhering, L.; Becker, S.; Rox, K.; Hilgenfeld, R. Crystal structure of SARS-CoV-2 main protease provides a basis for design of improved $\alpha$-ketoamide inhibitors. Science 2020, 368, 409-412. [CrossRef]

12. Amin, S.A.; Banerjee, S.; Gayen, S.; Jha, T. Protease targeted COVID-19 drug discovery: What we have learned from the past SARS-CoV inhibitors? Eur. J. Med. Chem. 2021, 113294. [CrossRef]

13. Steuten, K.; Kim, H.; Widen, J.C.; Babin, B.M.; Onguka, O.; Lovell, S.; Bolgi, O.; Cerikan, B.; Neufeldt, C.J.; Cortese, M. Challenges for targeting SARS-CoV-2 proteases as a therapeutic strategy for COVID-19. ACS Infect. Dis. 2021. [CrossRef] [PubMed]

14. Beck, B.R.; Shin, B.; Choi, Y.; Park, S.; Kang, K. Predicting commercially available antiviral drugs that may act on the novel coronavirus (SARS-CoV-2) through a drug-target interaction deep learning model. Comput. Struct. Biotechnol. J. 2020. [CrossRef] [PubMed]

15. Qamar, M.T.U.; Alqahtani, S.M.; Alamri, M.A.; Chen, L.-L. Structural basis of SARS-CoV-2 3CLpro and anti-COVID-19 drug discovery from medicinal plants. J. Pharm. Anal. 2020, 10, 313-319. [CrossRef] 
16. Gomez, C.R.; Espinoza, I.; Faruke, F.S.; Hasan, M.; Rahman, K.M.; Walker, L.A.; Muhammad, I. Therapeutic intervention of COVID-19 by natural products: A population-specific survey directed approach. Molecules 2021, 26, 1191. [CrossRef] [PubMed]

17. Wang, J. Fast Identification of Possible Drug Treatment of Coronavirus Disease-19 (COVID-19) through Computational Drug Repurposing Study. J. Chem. Inf. Model. 2020, 60, 3277-3286. [CrossRef]

18. Chang, Y.-C.; Tung, Y.-A.; Lee, K.-H.; Chen, T.-F.; Hsiao, Y.-C.; Chang, H.-C.; Hsieh, T.-T.; Su, C.-H.; Wang, S.-S.; Yu, J.-Y. Potential therapeutic agents for COVID-19 based on the analysis of protease and RNA polymerase docking. Preprints 2020. [CrossRef]

19. Terrier, O.; Dilly, S.; Pizzorno, A.; Chalupska, D.; Humpolickova, J.; Bouřa, E.; Berenbaum, F.; Quideau, S.; Lina, B.; Fève, B. Antiviral Properties of the NSAID Drug Naproxen Targeting the Nucleoprotein of SARS-CoV-2 Coronavirus. Molecules 2021, 26, 2593. [CrossRef]

20. Le, N.P.K.; Herz, C.; Gomes, J.V.D.; Förster, N.; Antoniadou, K.; Mittermeier-Kleßinger, V.K.; Mewis, I.; Dawid, C.; Ulrichs, C.; Lamy, E. Comparative Anti-Inflammatory Effects of Salix Cortex Extracts and Acetylsalicylic Acid in SARS-CoV-2 Peptide and LPS-Activated Human In Vitro Systems. Int. J. Mol. Sci. 2021, 22, 6766. [CrossRef]

21. Akhter, S.; Batool, A.I.; Selamoglu, Z.; Sevindik, M.; Eman, R.; Mustaqeem, M.; Akram, M.S.; Kanwal, F.; Lu, C.; Aslam, M. Effectiveness of Natural Antioxidants against SARS-CoV-2? Insights from the In-Silico World. Antibiotics 2021, 10, 1011.

22. Wu, C.; Liu, Y.; Yang, Y.; Zhang, P.; Zhong, W.; Wang, Y.; Wang, Q.; Xu, Y.; Li, M.; Li, X. Analysis of therapeutic targets for SARS-CoV-2 and discovery of potential drugs by computational methods. Acta Pharm. Sin. B 2020. [CrossRef]

23. Elzupir, A.O. Inhibition of SARS-CoV-2 main protease 3CLpro by means of $\alpha$-ketoamide and pyridone-containing pharmaceuticals using in silico molecular docking. J. Mol. Struct. 2020, 1222, 128878. [CrossRef]

24. Pettersen, E.F.; Goddard, T.D.; Huang, C.C.; Couch, G.S.; Greenblatt, D.M.; Meng, E.C.; Ferrin, T.E. UCSF Chimera-A visualization system for exploratory research and analysis. J. Comput. Chem. 2004, 25, 1605-1612. [CrossRef] [PubMed]

25. Wang, J.; Wang, W.; Kollman, P.A.; Case, D.A. Automatic atom type and bond type perception in molecular mechanical calculations. J. Mol. Graph. Model. 2006, 25, 247-260. [CrossRef] [PubMed]

26. O'Boyle, N.M.; Banck, M.; James, C.A.; Morley, C.; Vandermeersch, T.; Hutchison, G.R. Open Babel: An open chemical toolbox. J. Cheminform. 2011, 3, 1-14. [CrossRef] [PubMed]

27. Shapovalov, M.V.; Dunbrack Jr, R.L. A smoothed backbone-dependent rotamer library for proteins derived from adaptive kernel density estimates and regressions. Structure 2011, 19, 844-858. [CrossRef]

28. Trott, O.; Olson, A.J. AutoDock Vina: Improving the speed and accuracy of docking with a new scoring function, efficient optimization, and multithreading. J. Comput. Chem. 2010, 31, 455-461. [CrossRef]

29. Elzupir, A.O. Caffeine and caffeine-containing pharmaceuticals as promising inhibitors for 3-chymotrypsin-like protease of SARS-CoV-2. J. Biomol. Struct. Dyn. 2020, 1-8. [CrossRef]

30. Da Silva, A.W.S.; Vranken, W.F. ACPYPE-Antechamber python parser interface. BMC Res. Notes 2012, 5, 1-8. [CrossRef]

31. Wang, J.; Wolf, R.M.; Caldwell, J.W.; Kollman, P.A.; Case, D.A. Development and testing of a general amber force field. J. Comput. Chem. 2004, 25, 1157-1174. [CrossRef]

32. Maier, J.A.; Martinez, C.; Kasavajhala, K.; Wickstrom, L.; Hauser, K.E.; Simmerling, C. ff14SB: Improving the accuracy of protein side chain and backbone parameters from ff99SB. J. Chem. Theory Comput. 2015, 11, 3696-3713. [CrossRef]

33. Jorgensen, W.L.; Chandrasekhar, J.; Madura, J.D.; Impey, R.W.; Klein, M.L. Comparison of simple potential functions for simulating liquid water. J. Chem. Phys. 1983, 79, 926-935. [CrossRef]

34. Nelson, M.T.; Humphrey, W.; Gursoy, A.; Dalke, A.; Kalé, L.V.; Skeel, R.D.; Schulten, K. NAMD: A parallel, object-oriented molecular dynamics program. Int. J. Supercomput. Appl. High Perform. Comput. 1996, 10, 251-268. [CrossRef]

35. De Leeuw, S.W.; Perram, J.W.; Smith, E.R. Simulation of electrostatic systems in periodic boundary conditions. I. Lattice sums and dielectric constants. Proc. R. Soc. Lond. A Math. Phys. Sci. 1980, 373, 27-56.

36. Essmann, U.; Perera, L.; Berkowitz, M.L.; Darden, T.; Lee, H.; Pedersen, L.G. A smooth particle mesh Ewald method. J. Chem. Phys. 1995, 103, 8577-8593. [CrossRef]

37. Humphrey, W.; Dalke, A.; Schulten, K. VMD: Visual molecular dynamics. J. Mol. Graph. 1996, 14, 33-38. [CrossRef]

38. Miller, B.R., III; McGee, T.D., Jr.; Swails, J.M.; Homeyer, N.; Gohlke, H.; Roitberg, A.E. MMPBSA. py: An efficient program for end-state free energy calculations. J. Chem. Theory Comput. 2012, 8, 3314-3321. [CrossRef]

39. Roe, D.R.; Cheatham, T.E., III. PTRAJ and CPPTRAJ: Software for processing and analysis of molecular dynamics trajectory data. J. Chem. Theory Comput. 2013, 9, 3084-3095. [CrossRef] [PubMed]

40. Numata, J.; Wan, M.; Knapp, E.-W. Conformational entropy of biomolecules: Beyond the quasi-harmonic approximation. Genome Inform. 2007, 18, 192-205. [PubMed]

41. Lim, L.; Shi, J.; Mu, Y.; Song, J. Dynamically-driven enhancement of the catalytic machinery of the SARS 3C-like protease by the S284-T285-I286/A mutations on the extra domain. PLoS ONE 2014, 9, e101941. [CrossRef] [PubMed]

42. Saver, J.L. Citicoline: Update on a promising and widely available agent for neuroprotection and neurorepair. Rev. Neurol. Dis. 2008, 5, 167-177.

43. Dinsdale, J.; Griffiths, G.; Rowlands, C.; Castelló, J.; Ortiz, J.; Maddock, J.; Aylward, M. Pharmacokinetics of 14C CDP-choline. Arzneim. Forsch. 1983, 33, 1066-1070.

44. Anwar, M.U.; Adnan, F.; Abro, A.; Khan, M.R.; Rehman, A.U.; Osama, M.; Javed, S.; Baig, A.; Shabbir, M.R.; Assir, M.Z. Combined Deep Learning and Molecular Docking Simulations Approach Identifies Potentially Effective FDA Approved Drugs for Repurposing Against SARS-CoV-2. Comput. Biol. Med. 2021. [CrossRef] 
45. Prajapat, M.; Shekhar, N.; Sarma, P.; Avti, P.; Singh, S.; Kaur, H.; Bhattacharyya, A.; Kumar, S.; Sharma, S.; Prakash, A. Virtual screening and molecular dynamics study of approved drugs as inhibitors of spike protein S1 domain and ACE2 interaction in SARS-CoV-2. J. Mol. Graph. Model. 2020, 101, 107716. [CrossRef]

46. Elfiky, A.A. Ribavirin, Remdesivir, Sofosbuvir, Galidesivir, and Tenofovir against SARS-CoV-2 RNA dependent RNA polymerase (RdRp): A molecular docking study. Life Sci. 2020, 253, 117592. [CrossRef]

47. Chien, M.; Anderson, T.K.; Jockusch, S.; Tao, C.; Li, X.; Kumar, S.; Russo, J.J.; Kirchdoerfer, R.N.; Ju, J. Nucleotide analogues as inhibitors of SARS-CoV-2 polymerase, a key drug target for COVID-19. J. Proteome Res. 2020, 19, 4690-4697. [CrossRef]

48. Nar, H.; Schnapp, G.; Hucke, O.; Hardman, T.C.; Klein, T. Action of dipeptidyl peptidase-4 inhibitors on SARS-CoV-2 main protease. ChemMedChem 2021, 16, 1425-1426. [CrossRef]

49. Zhou, L.; Wang, J.; Liu, G.; Lu, Q.; Dong, R.; Tian, G.; Yang, J.; Peng, L. Probing antiviral drugs against SARS-CoV-2 through virus-drug association prediction based on the KATZ method. Genomics 2020, 112, 4427-4434. [CrossRef]

50. Zhang, Y.-N.; Zhang, Q.-Y.; Li, X.-D.; Xiong, J.; Xiao, S.-Q.; Wang, Z.; Zhang, Z.-R.; Deng, C.-L.; Yang, X.-L.; Wei, H.-P. Gemcitabine, lycorine and oxysophoridine inhibit novel coronavirus (SARS-CoV-2) in cell culture. Emerg. Microbes Infect. 2020, 9, $1170-1173$. [CrossRef] [PubMed]

51. Zheng, Z.; Groaz, E.; Snoeck, R.; De Jonghe, S.; Herdewijn, P.; Andrei, G. Influence of 4'-Substitution on the Activity of Gemcitabine and Its ProTide against VZV and SARS-CoV-2. ACS Med. Chem. Lett. 2020, 12, 88-92. [CrossRef] [PubMed]

52. Verma, A.K.; Aggarwal, R. Repurposing potential of FDA-approved and investigational drugs for COVID-19 targeting SARSCoV-2 spike and main protease and validation by machine learning algorithm. Chem. Biol. Drug Des. 2021, 97, 836-853. [CrossRef] [PubMed]

53. Borbone, N.; Piccialli, G.; Roviello, G.N.; Oliviero, G. Nucleoside analogs and nucleoside precursors as drugs in the fight against SARS-CoV-2 and other coronaviruses. Molecules 2021, 26, 986. [CrossRef]

54. Jo, S.; Kim, S.; Yoo, J.; Kim, M.-S.; Shin, D.H. A Study of 3CLpros as Promising Targets against SARS-CoV and SARS-CoV-2. Microorganisms 2021, 9, 756. [CrossRef] [PubMed]

55. Pillaiyar, T.; Meenakshisundaram, S.; Manickam, M. Recent discovery and development of inhibitors targeting coronaviruses. Drug Discov. Today 2020, 25, 668-688. [CrossRef]

56. Maurya, S.K.; Maurya, A.K.; Mishra, N.; Siddique, H.R. Virtual screening, ADME/T, and binding free energy analysis of anti-viral, anti-protease, and anti-infectious compounds against NSP10/NSP16 methyltransferase and main protease of SARS CoV-2. J. Recept. Signal Transduct. 2020, 40, 605-612. [CrossRef]

57. Kandeel, M.; Al-Nazawi, M. Virtual screening and repurposing of FDA approved drugs against COVID-19 main protease. Life Sci. 2020, 251, 117627. [CrossRef]

58. Choi, R.; Zhou, M.; Shek, R.; Wilson, J.W.; Tillery, L.; Craig, J.K.; Salukhe, I.A.; Hickson, S.E.; Kumar, N.; James, R.M. Highthroughput screening of the ReFRAME, Pandemic Box, and COVID Box drug repurposing libraries against SARS-CoV-2 nsp15 endoribonuclease to identify small-molecule inhibitors of viral activity. PLoS ONE 2021, 16, e0250019. [CrossRef]

59. Sharanya, C.; Abhithaj, J.; Sadasivan, C. Drug repurposing to identify therapeutics against COVID 19 with SARS-Cov-2 spike glycoprotein and main protease as targets: An in silico study. ChemRxiv 2020. [CrossRef]

60. Fadlalla, M. COVID19 Approved Drug Repurposing: Pocket Similarity Approach. ChemRxiv 2020. [CrossRef]

61. Rahman, N.; Basharat, Z.; Yousuf, M.; Castaldo, G.; Rastrelli, L.; Khan, H. Virtual screening of natural products against type II transmembrane serine protease (TMPRSS2), the priming agent of coronavirus 2 (SARS-CoV-2). Molecules 2020, $25,2271$. [CrossRef] [PubMed] 